\title{
On Resource Reuse for Cellular Networks with Femto- and Macrocell Coexistence
}

\author{
Yongsheng Shi, Allen B. MacKenzie, Luiz A. DaSilva \\ Bradley Department of Electrical and Computer Engineering \\ Virginia Tech, Blacksburg, Virginia \\ Email: \{shiys, mackenab, ldasilva\}@vt.edu
}

\author{
Kaveh Ghaboosi, Matti Latva-aho \\ Centre for Wireless Communications \\ University of Oulu, Oulu, Finland \\ Email: \{kavehg,matla\}@ee.oulu.fi
}

\begin{abstract}
This paper studies downlink resource reuse schemes for cellular networks with coexisting femtocells and macrocells. We examine two reuse schemes, termed split reuse and shared reuse. In this paper, we develop an analytical model of resource allocation based on random graphs. In our model, arbitrarily chosen communication links interfere with each other with a certain probability, which depends upon whether the links belong to femtocell or macrocell users. Using this model, we establish asymptotic bounds on the minimum number of resource blocks required to make an interference-free resource assignment for all the users in the network for large numbers of users. We assess these bounds using a simple greedy resource allocation algorithm to demonstrate that the bounds are reasonable in finite networks of plausible size. By applying the bounds, we establish the expected impact of femtocell networks on macrocell resource allocation under a wide variety of interference scenarios. Further, we assess the efficiency loss associated with split reuse, as an aid to determining whether resource allocators should use the simpler split reuse scheme or attempt to tackle the complexity and overhead associated with shared reuse.
\end{abstract}

\section{INTRODUCTION}

\section{A. Background}

Cellular networks have been a tremendous commercial success. By far the most effective way to improve overall system capacity has been to increase spatial reuse by making cells as small as possible. Deploying more operator-owned base stations (BSs) is one approach to reducing cell sizes, but it comes at a high cost. In addition, for many indoor users, the increased proximity to a BS may still not be sufficient to obtain high data rate service. Another approach to improving the capacity and indoor coverage of a cellular system is by introducing small overlay cells, called femtocells, into larger coverage area macrocells. A femtocell BS (fBS) is a small and inexpensive cellular BS with backhaul provided by a broadband access network, such as DSL or cable modem. Femtocells allow the cellular operators to improve indoor service coverage without much additional cost.

Despite the advantages of femtocells, there are some challenges associated with them. One issue is interference management. The macrocell BS is operator-owned and maintained, while the fBS may be customer-owned and is usually deployed in an ad-hoc fashion. In some areas, hundreds of fBSs may be deployed within the coverage zone of a single macrocell. Such deployment makes interference scenarios complicated. Femtocells need either coordination with surrounding macrocells or

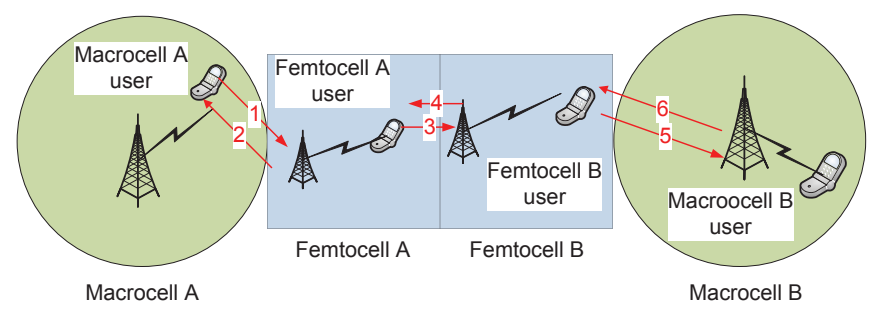

Fig. 1: Typical interference scenarios between femtocell users and macrocell users.

self-configuration to avoid severe interference to the macrocell users, while maintaining the required services to the femtocell users. Figure 1 shows potential interference scenarios between femtocell users and macrocell users. This paper focuses on coexistence between macrocells and femtocells and considers the downlink side using Orthogonal Frequency-Division Multiple Access (OFDMA). We use the term resource block to represent the minimal allocation unit for one user; this term is also used in the Long Term Evolution (LTE) standard. A resource block is translated to a number of consecutive OFDM symbols in the time domain and a number of consecutive subcarriers in the frequency domain. Any two users served by the same BS cannot use the same resource block in the downlink.

In our work, we consider two commonly used methods for resource reuse, as illustrated in Figure 2. In split reuse, shown in Figure 2(a), the resources are separated such that some of the resource blocks are solely used by femtocell users and the remaining resource blocks are only for the macrocell users. In shared reuse, shown in Figure 2(b), macrocells are able to access all the resource blocks while femtocells are permitted to access only some of the resource blocks. Split reuse reduces the complexity of the resource allocation algorithm but lowers resource reuse efficiency, while shared reuse is expected to result in better performance but may require a more complex algorithm to handle interference between femtocell and macrocell users.

\section{B. Related Work}

The authors of [1] provide a survey of femtocells, comparing several different ways to extend network coverage and enhance service quality in terms of capacity gain, coverage 


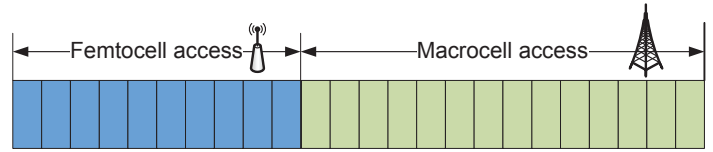

(a) Case I: Split Reuse

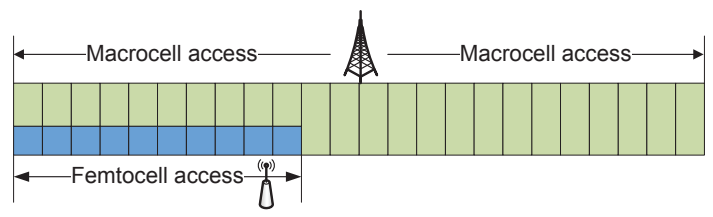

(b) Case II: Shared Reuse

Fig. 2: Resource allocation mechanisms for OFDMA-based femtocell networks.

area, indoor coverage, cost, and operator benefit. They also describe several key technical challenges, including spectrum allocation, timing and synchronization, femtocell access policies, and network architecture. Research directions in interference management are also presented.

The majority of work on channel allocation for OFDMAbased cellular networks focuses on macrocells and proposes various methods to mitigate inter-macrocell interference with frequency reuse factor of 1 [2]-[4]. The study of interference management in femtocell deployment is a recent research area. In [5] and [6], Claussen and Ho study co-channel interference management for CDMA-based femtocells and macrocells. Simulations are performed for a residential femtocell scenario to determine the potential effects on macrocell users with and without self-provisioning of femtocell power. The results show that the increase in call drop probabilities due to the deployment of these femtocells would be low if power adaptation techniques were implemented, but unacceptably high otherwise. The work also shows that, despite low transmit power, the short distance between the fBS and the femtocell user results in high achievable femtocell throughput for both uplink and downlink in most covered femtocell areas.

In the context of OFDMA-based systems, López-Pérez et al. [7] provide and interference analysis and some guidelines on how the spectrum allocation and interference mitigation problems can be approached. Self-configuration and selfoptimization techniques are used for interference avoidance. In addition, these authors study how to share the resources and avoid interference for different femtocell access mechanisms, namely open access, closed access, and hybrid access in [8]. The authors of [9] address optimal resource allocation between macrocells and femtocells. They also propose a location-based resource management solution for reaching maximal spatial reuse by femtocells. The work in [10] presents a procedure for performance evaluation of a multi-carrier cellular system with femtocells using the same carrier as the macrocells.

\section{Our Contributions}

For macrocell/femtocell networks, the above related work either provides guidelines on resource reuse, or addresses optimum resource allocation under various assumptions, but does not address the following basic questions: Without significant degradation to existing users' performance, what is the impact on resource allocation of introducing femtocells into macrocells with respect to both split reuse and shared reuse? How many additional resource blocks are required to accommodate femtocells under different resource reuse schemes? Our paper addresses these questions and makes the following contributions:

- By applying a random conflict graph model, we analyze properties of the different reuse schemes and derive upper and lower bounds on the minimum number of resource blocks required for the network.

- We develop a simple heuristic algorithm for resource allocation between femtocell and macrocell users. In the majority of cases, the heuristic algorithm results tightly track the upper bound derived in split reuse and the lower bound in shared reuse.

\section{SySTEM MODEL}

We formulate our problem using the protocol model [11] of interference. Although less expressive than a Signal-toInterference-plus-Noise Ratio (SINR) model, the protocol model allows us to study resource allocation analytically. Under this model, interference is a binary condition: either a pair of links interfere with each other or they do not.

\section{A. Conflict Graph}

A conflict graph is a graph $G$ with vertex set $V$ and edge set $E$. Each vertex $v \in V$ in the conflict graph represents a communication link in the network. Two vertices $u$ and $v$ are connected by a non-directional edge $(u, v) \in E$ if and only if their associated communication links will interfere with each other. In other words, the communication links represented by two adjacent vertices in the conflict graph cannot share the same resource block.

A conflict graph is shown in Figure 3. Figure 3(a) illustrates communication activities in a macrocell/femtocell network while figure 3(b) shows the corresponding conflict graph. Macrocell user downlinks 1, 2 and 3 naturally interfere with each other and therefore their corresponding nodes in the conflict graph are connected. Femtocell user links 4 and 5 are close enough to interfere with each other. Links 2 interferes with femtocell links 4 and 5 (due to the long distance from the macrocell BS to mobile user 2). Link 2 also interferes with femotcell link 6 and link 3 interferes with femtocell links 4 and 5 (due to the proximity of the mobile users to the femtocells).

\section{B. Problem Formulation}

We focus on the downlink to all femtocell and macrocell users within one macrocell in a cellular network. The macrocell has $M$ macrocell users and $F$ femtocells inside. Each femtocell has one active femtocell user. We assign one user 


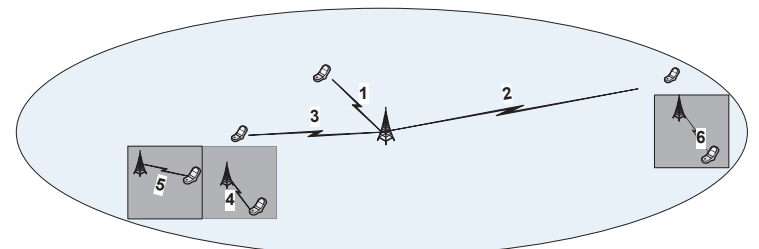

(a) A Network with a mix of macrocell and femtocells.

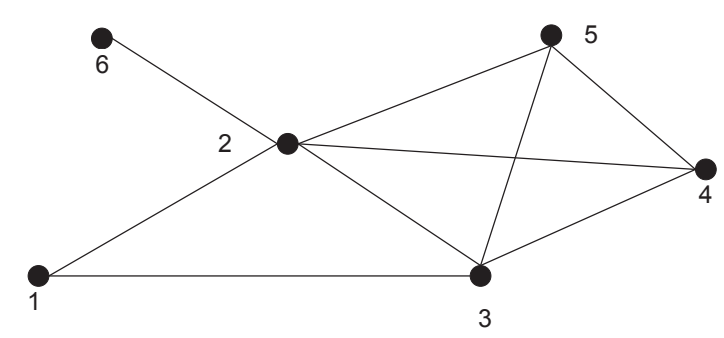

(b) Conflict graph based on network scenario in (a).

Fig. 3: An example of conflict graph.

to each femtocell for convenience and ease of analysis; our model is easily adapted to an arbitrary number of users in each femtocell by modifying the conflict graph accordingly. The number of available resource blocks is $N$.

Based on the constructed conflict graph, the resource allocation problem is to build an $(M+F) N$ binary resource assignment matrix $\mathbf{A}$. An element in $\mathbf{A}, a_{i, k}$, is 1 when resource block $k$ is assigned to link $i$ and 0 otherwise. Two links $i$ and $j$ cannot use the same resource block if they conflict, that is if $(i, j) \in E$. A resource assignment matrix A that satisfies this condition is feasible and represents an interference-free resource assignment, i.e. $a_{i, k} \times a_{j, k}=0$ if $(i, j) \in E$. We denote the set of feasible resource assignment matrices as $\mathcal{A}$.

For the purpose of analysis, we adopt random graphs to construct sample conflict graphs. Four parameters are needed to construct such a random conflict graph: $M$ (the number of macrocell users), $F$ (the number of femtocell users), $p_{f f}$ (the probablity that two arbitrarily chosen femtocell links interfere) and $p_{m f}$ (the probablity that an arbitrarily chosen femtocell link interferes with an arbitrarily chosen macrocell link). The $M$ vertices representing the macrocell links are fully connected because a resource block cannot be shared by two macrocell users in the same macrocell.

In split reuse, there will be no resource conflicts between macrocell and femtocell users. In that case, we can focus on the subgraphs $G_{m}$, which consists of $M$ vertices that are fully connected, and $G_{f}$, which is a Bernoulli graph with $F$ vertices and connection probablity $p_{f f}$. In shared reuse, beyond the three parameters in split reuse, we need to consider connection probablity, $p_{m f}$, between vertices in $G_{m}$ and $G_{f}$. We will obtain the minimum number of resource blocks to support full network connectivity in both reuse schemes.

\section{Problem Analysis}

\section{A. Graph Theory Preliminaries}

It is useful to introduce some basics of graph theory before we proceed with the resource allocation problem.

Any feasible matrix $\mathbf{A} \in \mathcal{A}$ results in conflict free transmission for all links in the network. Such an $\mathbf{A}$ assigns different resource blocks to any adjacent vertices in the conflict graph $G$. This motivates us to apply graph coloring to analyze this problem. Graph coloring tries to find the minimum number of colors that can color a graph such that any adjacent vertices are assigned different colors. The following definitions and propositions will be useful:

Definition 1: The chromatic number of a graph $G$, written $\chi(G)$, is the minimum number of colors needed to label the vertices so that adjacent vertices receive different colors [12].

Definition 2: A clique in a graph is a set of fully connected vertices. An independent set in a graph is a set of vertices that are not connected to each other; $S \subseteq V$ is an independent set if there do not exist $u, v \in S$ such that $(u, v) \in E$ [12].

Definition 3: The clique number of a graph $G, \omega(G)$, is the maximum size of a set of fully connected vertices (clique) in $G$. The independence number of a graph $G, \beta(G)$, is the maximum size of an independent set of vertices [12].

We use the model $\mathscr{G}\{n, p\}$ to describe Bernoulli random graphs, where $0<p<1$. The model consists of all graphs with vertex set $V=\{1,2, \ldots, n\}$ such that the probablity that a particular graph with edge set $E$ is observed is $p^{|E|}(1-$ $p)^{(n(n-1) / 2)-|E|}$. That is, edges are chosen independently with probablity $p$.

Definition 4: We shall say that almost every (a.e.) random graph with probablity $p$ has a certain property $\mathcal{Q}$ if $P(\mathcal{Q}) \rightarrow 1$ as $n \rightarrow \infty$ under $\mathscr{G}\{n, p\}$ [13].

\section{B. Random Model}

Most graph properties of random graphs, such as clique number, independence number, and chromatic number, are based on the concept of almost every because the graph properties are not deterministic. In a conflict graph generated for femtocell users, the number of vertices is not infinite, but for a typical case, the number of femtocell users may easily reach hundreds. Thus we believe applying properties derived from the random graph model is reasonable.

To find the lower and upper bounds on the chromatic number of a random graph, we begin by determining the independence number. For $r \in \mathbb{N}$, let $X_{r}$ denote the number of independent sets with $r$ vertices of a graph $G$. Let $Y$ be the independence number of $G$. By definition $Y=\max \{r$ : $\left.X_{r}>0\right\}$.

If $G$ is chosen according to $\mathscr{G}\{n, p\}$ then $X_{r}$ is a random variable. For any interger $r$, the expected number of independent sets of size $r$ in $G$ is

$$
E\left(X_{r}\right)=\left(\begin{array}{l}
n \\
r
\end{array}\right)(1-p)^{r(r-1) / 2} .
$$

According to [13], for small values of $r$ and $n$ sufficiently large, $E\left(X_{r}\right)$ is large and the variance is fairly small. As the 
value of $r$ increases, $E\left(X_{r}\right)$ drops below 1 rather suddenly. For some $r_{0}$, we have $E\left(X_{r_{0}}\right) \gg 1$ and $E\left(X_{r_{0}+1}\right) \ll 1$. We are interested in this value of $r_{0}$, as we have $Y=r_{0}$ with high probability.

We can re-write Equation 1 using Stirling's approximation:

$$
n ! \approx \sqrt{2 \pi n}\left(\frac{n}{e}\right)^{n}
$$

This yields

$$
E\left(X_{r}\right) \approx \frac{n^{n+\frac{1}{2}}(n-r)^{-n+r-\frac{1}{2}} r^{-r-\frac{1}{2}}(1-p)^{\frac{r(r-1)}{2}}}{\sqrt{2 \pi}} .
$$

Notice that if we could find a real number $\bar{r}_{0} \geq 2$ that makes the right hand side of Equation 2 equal to 1, then we would expect $Y=r_{0}=\left\lfloor\bar{r}_{0}\right\rfloor$ with high probability (again for sufficiently large $n$ ). Matula solved an equivalent problem in [14], finding an approximate solution, which we denote $\hat{r}_{0}$,

$$
\hat{r}_{0}=2 \log _{b} n-2 \log _{b} \log _{b} n+2 \log _{b}\left(\frac{e}{2}\right)+1
$$

where $b=1 /(1-p)$.

In any proper graph coloring, every color class must be an independent set. Every color class thus contains at most roughly $\hat{r}_{0}$ vertices, resulting in following lower bound for chromatic number of a.e. $G$ :

$$
\chi(G) \geq \frac{n}{\hat{r}_{0}} .
$$

An upper bound on the chromatic number of $G$ is more difficult to obtain. The best bound we can find is from [15] and is given by

$$
\chi(G) \leq \frac{n}{2 \log _{b} n}\left(1+\frac{3 \log \log n}{\log n}\right) .
$$

Combining Equation 4 and 5 and considering $\hat{r}_{0} \leq 2 \log _{b} n$ (according to Equation 3 while $\mathrm{n}$ is sufficiently large), we have the following bounds for the chromatic number for a.e. $G$ :

$$
\frac{n}{\hat{r}_{0}} \leq \chi(G) \leq \frac{n}{\hat{r}_{0}}\left(1+\frac{3 \log \log n}{\log n}\right) .
$$

Note that as $n \rightarrow \infty$, the lower and upper bounds in Equation 6 converge. This is $\chi(G) \rightarrow n / \hat{r}_{0}$ for a.e. $G$.

1) Split Reuse of Resource: Now we map the random graph model to split reuse of resource blocks. The lower bound and upper bound of the number of resource blocks required to serve $F$ femtocell users (the random graph $G_{f}$ ) are

$$
\frac{F}{\hat{r}_{0}} \leq \chi\left(G_{f}\right) \leq \frac{F}{r_{0}^{\prime}}\left(1+\frac{3 \log \log F}{\log F}\right)
$$

where

$$
\begin{aligned}
\hat{r}_{0} & =\max \left(2,2 \log _{b} F-2 \log _{b} \log _{b} F+2 \log _{b}\left(\frac{e}{2}\right)+1\right) \\
b & =1 /\left(1-p_{f f}\right) .
\end{aligned}
$$

Let us denote the lower bound and the upper bound as $\chi_{\text {lower }}\left(G_{f}\right)$ and $\chi_{\text {upper }}\left(G_{f}\right)$. Obviously, the minimum number of resource blocks required to guarantee no macrocell user outage is $M$ and the number of resource blocks for femtocell users is roughly $N_{f}=\chi_{\text {upper }}\left(G_{f}\right)$. Hence the total number of resource blocks required for whole network is $N_{\min }=$ $M+N_{f}$.

2) Shared Reuse of Resource: Although it is challenging to allocate the resources to users, a possible benefit of shared reuse is that this scheme potentially requires fewer resource blocks to support the same size network or, from another prospective, is able to serve more users with the same amount of resources. In the shared reuse scheme, another factor that needs to be considered is where to split the resource blocks such that part of them are shared by macrocell and femtocell users, and part of them is exclusively for macrocell users. Clearly, sharing all resources among all users allows for the most efficient allocation. This would be the case where the split point in Figure 2(b) is at the far right, a reuse scheme known as "universal reuse." In this section, we focus on the analysis of such a universal reuse scenario. Other locations of the split point may result in slightly less efficient results.

In a random graph model with $M$ macrocell users and $F$ femtocell users with interference parameters $p_{f f}$ and $p_{m f}$, under shared reuse of resources the minimum number of required resource blocks, $N_{\min }$, follows:

$$
\max \left\{M, N_{f}\right\} \leq N_{\min } \leq M+N_{f}
$$

The left-hand part is easy to check. It is also intuitive that the right-hand part holds because graph $G$ is formed by graph $G_{m}$ and graph $G_{f}$. The worst case (most number of colors needed) of $G$ is that every vertex in $G_{m}$ connects to every vertex in $G_{f}$, i.e. $p_{m f}=1$. Thus any color used in $G_{m}$ cannot be reused in $G_{f}$ and the total number of colors for $G$ is at $M+N_{f}$.

This confirms that shared reuse of resource is more efficient than split reuse. However, the upper bound of $N_{\min }$ shown in equation 8 is pessimistic. We attempt to tighten this upper bound.

A macrocell user on average interferes with $F p_{m f}$ femtocell users. The extreme case is that all macrocell users interfere with the same set of $F p_{m f}$ femtocell users. In this case, all resource blocks used by macrocell users cannot be reused by this set of femtocell users. We would like to find an upper bound on the minimum number of resource blocks required for the set of $F p_{m f}$ femtocell users. Thus adding this number to $M$ can give a reasonable upper bound on total number of resource blocks required in shared reuse. By Equation 6, an upper bound on the minimum required number of resource blocks in shared reuse is

$$
N_{\min } \leq \max \left(M+\frac{F p_{m f}}{\hat{r}_{0}}\left(1+\frac{3 \log \log \left(F p_{m f}\right)}{\log \left(F p_{m f}\right)}\right), N_{f}\right)
$$




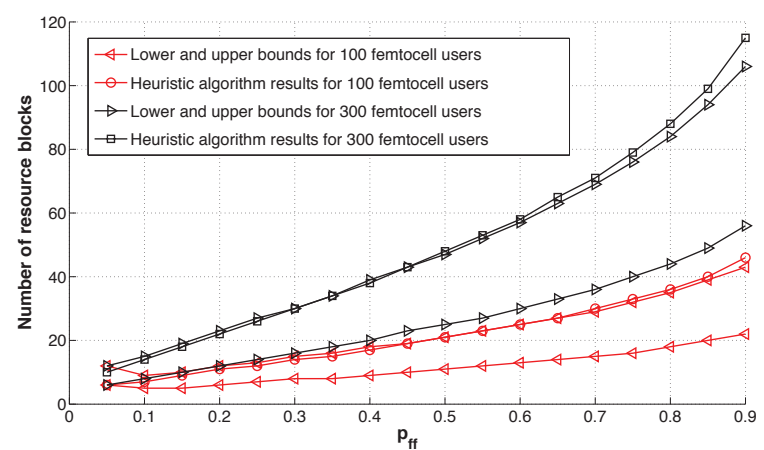

Fig. 4: The number of resource blocks for femtocell users.

where

$$
\begin{aligned}
\hat{r}_{0}= & \max \left(2,2 \log _{b}\left(F p_{m f}\right)-2 \log _{b} \log _{b}\left(F p_{m f}\right)\right. \\
& \left.+2 \log _{b}\left(\frac{e}{2}\right)+1\right) \\
b= & 1 /\left(1-p_{f f}\right) .
\end{aligned}
$$

\section{Numerical Results}

A single coloring algorithm can be used to solve the resource block allocation problem, i.e., for a given network, we find the minimum number of resource blocks required to serve all users. The optimal single coloring problem is known to be NP-hard and thus is intractable in a large scale network. In this section, we use a simple greedy algorithm to solve the resource allocation problem and compare results to the lower and upper bounds developed in the previous section. Given a conflict graph, the algorithm sorts all network links in descending order based on the number of conflicting links and marks each color with a number. The algorithm goes through the link list from the top, assigning each link to the eligible least numbered color based on the conflict scenario.

For split reuse, we are interested in the relationship between the lower and upper bounds and the heuristic algorithm results. Figure 4 shows the results. From this figure, the number of resource blocks required by the heuristic algorithm tracks the upper bound closely. The number from the heuristic algorithm is slightly larger than the upper bound when $p_{f f}$ is large. This can be explained in two ways. In our random model, all bounds are under the umbrella of almost every as $n \rightarrow \infty$. Though the number of nodes is large, it is not guaranteed that all graph realizations are covered by the upper bound. Second, the heuristic algorithm is not optimal. When $p_{f f}$ increases, the performance of the heuristic algorithm degrades fast. On the other hand, the figure suggests that when $p_{f f}$ is not large, the upper bound gives a reasonable estimate of the number of resource blocks required by the heuristic algorithm.

For shared reuse, we would like to compare the performance of the heuristic algorithm to our derived upper bound in Equation 9. There are four variables: $M, F, p_{f f}$, and $p_{m f}$. In practice, it is not likely that $p_{f f}$ and $p_{m f}$ are close to 1 or even larger than 0.5 . We try to use reasonable estimates of $p_{f f}$ and $p_{m f}$. We assume an inter-site (macrocell BS) distance

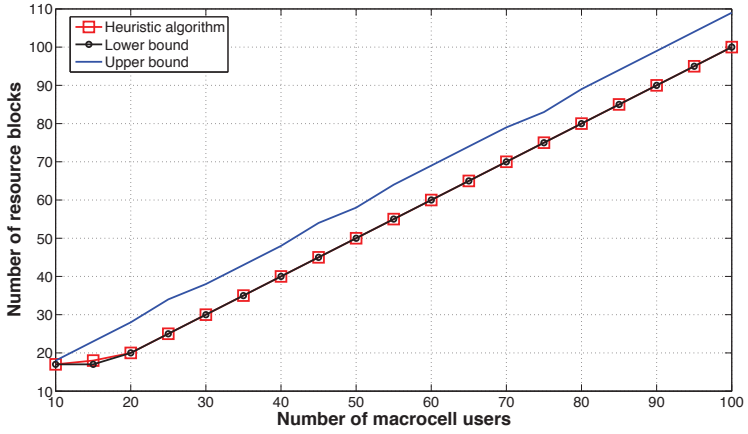

Fig. 5: The Number of resource blocks required in shared reuse with $p_{f f}$ of 0.2 .

of $1 \mathrm{~km}$ for dense urban areas. In this case, the area of a macrocell will be $0.289 \mathrm{~km}^{2}$. Population density in dense urban areas is 10,000 to 20,000 per $\mathrm{km}^{2}$. We take the upper bound of 20,000 and assume there are 7692 household units (2.6 persons per household) per $\mathrm{km}^{2}$. This corresponds to 2223 household units per macrocell. Furthermore, we assume cellular phone penetration of $80 \%$, operator penetration of $40 \%$, and femtocell penetration of $30 \%$. Thus we have approximately 213 femtocells in one macrocell located in dense urban areas. We randomly drop 200 femtocells in the coverage area of one macrocell. By applying widely used propagation models [16][18], $p_{f f}$ is about 0.2 on average and $p_{m f}$ ranges from 0.2 to 0.3 depending on the number of macrocell users.

Figure 5 shows the results using the above parameters. The heuristic algorithm results closely track the lower bound given by $\max \left\{M, N_{f}\right\}$. In fact, they are equal in most cases. This indicates that with a small $p_{m f}$ (light interference between macrocell users and femtocell users), the lower bound is tight. In such a case, most resource blocks used by macrocell users can be reused by femtocell users. We also drew some three-dimensional figures showing different values of $p_{m f}$ (not included in this paper). Those figures suggest that with a large $p_{m f}$ and a small $M$ (though this case is not likely in reality), the results of the heuristic algorithm closely track the upper bound. However, in any case, if $M$ is large enough, the lower bound is always a good estimate on the required number of resource blocks. In shared reuse, if $M$ is large, it is likely that the clique number is $M$. In [19], it is shown that in a graph, the chromatic number is at most 1.21 times the clique number.

We also use 200 femtocell users with 50 and 100 macrocell users as examples to show results of the required number of resource blocks for shared reuse at different $p_{m f}$ and $p_{f f}$, in Figures 6 and 7. Clearly, as the number of macrocell users increases, the number of resource blocks required for the whole network is dominated by the number of resource blocks required by the macrocell users, as long as fBSs use sufficiently low transmit power (possibly through power control) to insure that $p_{f f}$ and $p_{m f}$ are not exceptionally high. 


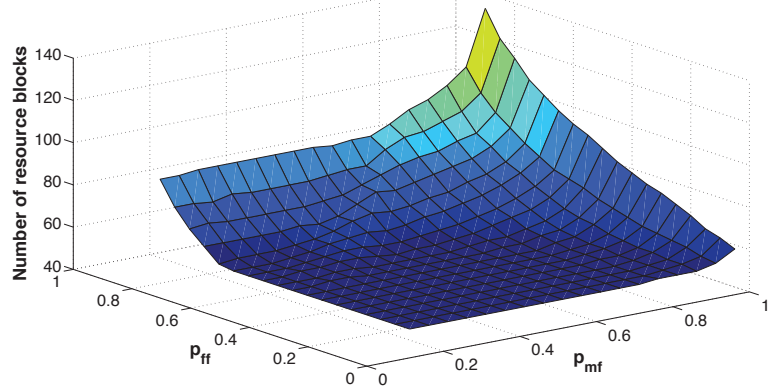

Fig. 6: The number of resource blocks for 200 femtocell users and 50 macrocell users.

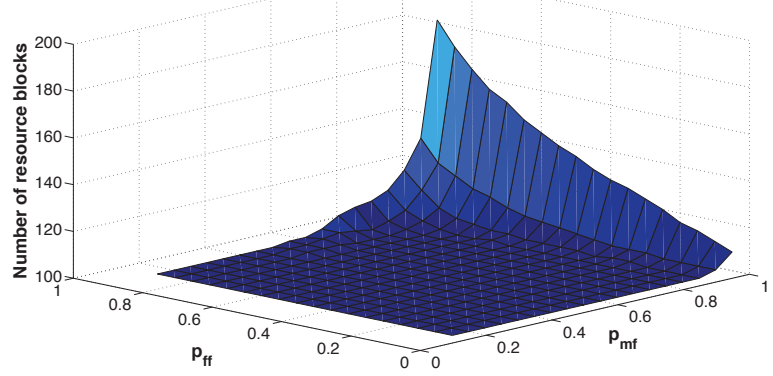

Fig. 7: The number of resource blocks for 200 femtocell users and 100 macrocell users.

\section{CONCLUSION}

In this paper, we have discussed some of the challenges of introducing femtocells coexistint with current cellular networks. We have used a protocol model and a random conflict graph to describe the spectrum reuse problem. We then applied random graph theory to analyze the problem, deriving upper and lower bounds on the number of resource blocks required to satisfy minimal assignment requirements for the network. We developed a heuristic algorithm to generate numerical results to compare against the theoretic bounds. For split reuse, the upper bound gives a good estimate on the number of resource blocks required. For shared reuse, we have concluded that with a small $p_{m f}$ (indicating light interference between macrocell users and femtocell users), which is expected in reality, the lower bound is tight. By applying the derived bounds and different values of $M, F, p_{f f}$, and $p_{m f}$, we have obtained estimates on the number of resource blocks required for the cellular networks with femtocells and macrocells, under different resource reuse schemes and network environments.

\section{REFERENCES}

[1] Vikram Chandrasekkhar, Jeffrey G. Andrews, and Alan Gatherer, "Femotocell Networks: A Survey," IEEE Communication Magazine, vol. 46, no. 9, pp. 59-67, Sept. 2008.

[2] Arne Simonsson, "Frequency Reuse and Intercell Interference CoOrdination in E-UTRA," Proceedings IEEE Vehicular Technology Conference (VTC), pp. 3091-3095, Apr. 2007.

[3] Bin Fan, Yu Qian, Kan Zheng and Wenbo Wang, "A Dynamic Resource Allocation Scheme Based on Soft Frequency Reuse of OFDMA Systems," Proceedings IEEE International Symposium on Microwave, Antenna, Propagation and EMC Technologies for Wireless Communications, pp. 1-4, Aug. 2007.

[4] Seok Ho Won, Hyeong Jun Park, James O. Neel and Jeffrey H. Reed, "Inter-Cell Interference Coordination/Avoidance for Frequency Reuse by Resource Scheduling in an OFDM-based Cellular System," Proceedings IEEE Vehicular Technology Conference (VTC), pp. 1722-1725, Oct. 2007.

[5] Lester T. W. Ho and Holger Claussen, "Effects of User-Deployed, CoChannel Femtocells on the Call Drop Probablity in a Residential Scenario," Proceedings IEEE International Symposium on Personal, Indoor and Mobile Radio Communications (PIMRC), Sept. 2007.

[6] Holger Claussen, "Performance of Macro-and Co-Channel Femtocells in a Hierarchical Cell Structure," Proceedings IEEE Internaional Symposium on Personal, Indoor and Mobile Radio Communications, Sept. 2007.

[7] David Lépez-Pérez, Alvaro Valcarce, Guillaume de la Roche, and Jie Zhang, "OFDMA Femtocells: A Roadmap on Interference Avoidance," IEEE Communication Magazine, vol. 47, no. 9, pp. 41-48, Sept. 2009.

[8] Guillaume de la Roche, Alvaro Valcarce, David Lépez-Pérez, and Jie Zhang, "Access Control Mechanisms for Femtocells," IEEE Communication Magazine, vol. 48, no. 1, pp. 33-39, Jan. 2010.

[9] Karthikeyan Sundaresan and Sampath Rangarajan, "Efficient Resource Management in OFDMA Femto Cells," Proceedings of the tenth ACM international symposium on Mobile ad hoc networking and computing (MobiHoc), pp. 33-42, May. 2009.

[10] Romeo Giuliano, Pierpaolo Loreti, Franco Mazzenga, and Giovanni Santella "Planning and Performance Evaluation of OFDM/OFDMA Multi-carrier Cellular Systems with Femto Cells," Proceedings of the 2009 International Conference on Wireless Communications and Mobile Computing: Connecting the World Wirelessly, pp. 1478-1482, 2009.

[11] P. Gupta and P. R. Kumar, "the capacity of wireless networks," IEEE Transactions on Information Theory, vol. 46, no. 2, pp. 388-404, 2000.

[12] Douglas B. West, Introduction to Graph Theory (2nd Edition), Prentice Hall, Upper Saddle River, NJ, United States, Sept. 2000.

[13] Bélla Bollobás, Random Graphs (2nd Edition), Cambridge University Press, Cambridge, United Kindom, Sept. 2001.

[14] David W. Matula, "The employee party problem," Notice of the American Mathematical Society, vol. 19, no. 7, pp. A382, Jul. 1972

[15] Colin McDiarmid, "On the chromatic number of random graphs," Random Structures and Algorithms, vol. 1, no. 4, pp. 435-442, Oct. 2006

[16] "3GPP Technical Specification Group Radio Access Networks; Radio Frequency (RF) system scenarios (Release 7)," 3GPP, 2007. [Online] Available at http://www.3gpp.org/ftp/Specs/archive/25_series/25. 942/25942-700.zip

[17] J.M. Keenan and A. J. Motley, "Radio coverage in buildings," British Telccom Technology, vol. 8, no. 1, pp. 19-24, 1990

[18] "3GPP Technical Specification Group Radio Access Networks; FDD Base Station (BS) classification (Release 7)," 3GPP, 2007. [Online] Available at http://www.3gpp.org/ftp/Specs/archive/25_series/25. 951/25951-700.zip

[19] Pradeepkumar Mani and David Petr, "Clique Number vs. Chromatic Number in Wireless Interference Graphs: Simulation Results," IEEE Communications Letters, vol. 11, no. 7, Jul. 2007 